Fatyga Kamil, Tarczyńska Marta, Gawęda Krzysztof, Mazur Rafal, Glodek Lukasz. Entesopathy of the lateral epicondyle of the humeral bone - current treatment options. Journal of Education, Health and Sport. 2020;10(3):140-147. eISSN 2391-8306. DOI http://dx.doi.org/10.12775/JEHS.2020.10.03.015

https://apcz.umk.pl/czasopisma/index.php/JEHS/article/view/JEHS.2020.10.03.015

https://zenodo.org/record/3730081

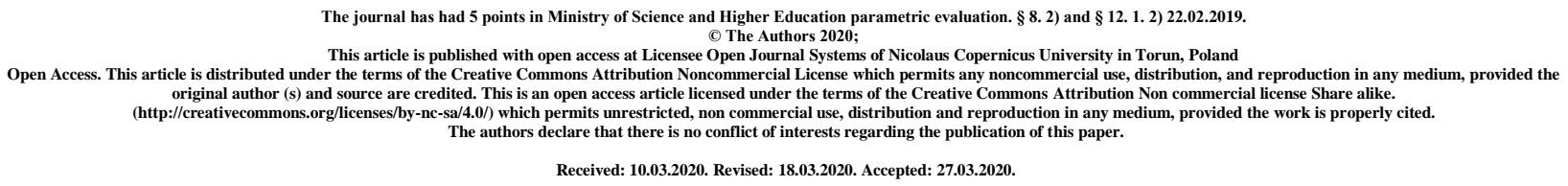

\title{
Entesopathy of the lateral epicondyle of the humeral bone - current treatment options
}

\author{
Kamil Fatyga $^{1}$, Marta Tarczyńska ${ }^{1}$, Krzysztof Gawęda ${ }^{1}$, Rafał Mazur $^{2}$, Lukasz Głodek ${ }^{1}$ \\ 1 Medical University of Lublin, Department of Orthopaedics and Traumatology, Lublin, \\ Poland \\ 2 Trauma and Orthopaedics Department, SPSK im. A. Grucy, Otwock, Poland
}

1) Kamil Fatyga M.D. ${ }^{1}$ : https://orcid.org/0000-0001-8319-4549 e-mail: kamil_fatyga@wp.pl

2) Marta Tarczyńska M.D. , Ph.D¹: http://orcid.org/0000-0002-5268-6230 e-mail:

martatarczyn@o2.pl

3) Krzysztof Gawęda M.D. Ph.D ${ }^{1}$ : http://orcid.org/0000-0002-1374-3343 e-mail: krzylub@o2.pl

4) Rafał Mazur M.D. ${ }^{2}$ : http://orcid.org/0000-0002-8486-5567, e-mail: rafall.mazur@gmail.com

5) Łukasz Głodek M.D. ${ }^{1}$ : http://orcid.org/0000-0002-3741-4392 e-mail:

lukaszglodek9@o2.pl

\begin{abstract}
Chronic pain located on the lateral surface of the elbow in the area of the lateral epicondyle of the humerus is characteristic for a disease called tennis elbow, which belongs to the group of enthesopathies, i. e. diseases related to bone tendon attachment. The cause of the disease are microinjuries caused by repetitive movements and chronic overloads. Degenerative changes occur in the tendons of the finger and wrist extensor muscles which attach to the lateral epicondyle of the humerus. The problem usually affects the extensor carpi radialis brevis muscle. A typical location for pain is the lateral side of the elbow joint but it can often radiate to the forearm and wrist. In our
\end{abstract}


paper we presented the most commonly used and most effective methods of treatment of the disease. The least invasive methods of physiotherapy and rehabilitation include home exercise, ultrasound and phonophoresis, cryotherapy, light therapy, laser therapy and radial shock wave terapy. We compared the efficacy of pharmacological methods such as non-steroidal antiinflammatory drugs, steroid injections with lidocaine, platelet-rich plasma, hyaluronic acid, botulinum toxin, collagen. We also mentioned the indications and surgical techniques of the tennis elbow.

Key words: medicine; orthopedics; entesopathy of the lateral epicondyle of the humeral bone; enthesopathy; injection; tennis elbow; treatment

\section{Introduction}

Chronic pain on the lateral surface of the elbow in the vicinity of the lateral epicondyle of the humerus is a common musculoskeletal presentation in primary care patients and patients of surgical and orthopedic clinics. This disease, commonly referred to as tennis elbow, belongs to the group of enthesopathies, i.e. disorders involving the attachment of a tendon to a bone. Tennis elbow is a disease of the finger and wrist extensor tendons which attach to the lateral epicondyle of the humerus. Most often, the condition affects the tendon of the extensor carpi radialis brevis muscle.

Tennis elbow is caused by microinjuries that occur as a consequence of repetitive movements and prolonged overload of the hand, wrist and forearm. The complaints are associated with degenerative changes to the tendons, most often resulting from abnormal blood supply to the point of tendon attachment to the bone. The lesions generally involve damage to the collagen fibers which are the building blocks of tendons.

The dominant subjective symptom of tennis elbow is pain on the outer lateral side of the elbow. The pain often radiates to the forearm and wrist. It usually increases when the patient tries to clench their fist or bend their wrist backward against resistance. Typically, there is tenderness on palpation that extends from the tendon attachment of wrist and finger extensor muscles to the lateral epicondyle of the humerus. Patients experience difficulties performing everyday activities, such as raising a glass or opening a door. The origin of the pain often seems difficult to pinpoint. Because soreness accompanies almost all activities of the hand which involve grasping or clenching, this suggests that it comes from flexor muscles. The pain, however, originates in the group of extensors since a hand's grasping function requires stabilization of the wrist by fixing it in dorsiflexion at $30^{\circ}$ $50^{\circ}$. To obtain such a static position, prolonged isotonic or variable tension of extensor muscles and tendons is needed.

Tennis elbow occurs in about 3\% of the human population each year. The disease was first described in tennis players, hence its name. Despite the name, tennis players constitute only $10 \%$ of all patients. The condition, which is often erroneously referred to as lateral epicondylitis (Epicondylitis lateralis), i.e. inflammation of the lateral epicondyle of the humeral bone, mainly affects people who engage daily in activities that involve repeated alternating extension and rotation of the wrist, i.e. movements which exert load on the attachment of wrist extensor muscles. Some authors put tennis elbow under an umbrella nosological category of tendino- and enthesopathies which subsumes diseases of the tendons and their attachments. They believe, based on epidemiological studies, that smoking is one of the risk factors for tennis elbow.

Cyriax and Winkel have distinguished five pathomorphic types of tennis elbow depending on which tendon is affected by the disease.

According to their classification, type I tennis elbow occurs when the disease involves the origin 
(proximal attachment) of the extensor carpi radialis longus muscle. Type II tennis elbow affects the origin of the extensor carpi radialis brevis muscle. Type III develops in the tendon body of the extensor carpi radialis brevis. Type IV is a lesion that is classified as a tendinopathy, but is not located at a tenoperiosteal junction but at the muscle belly of the extensor carpi radialis brevis. In type $\mathrm{V}$ tennis elbow, lesions develop at the origin of the extensor digitorum.

In the present study, we review the state of the art in the treatment of enthesopathy of the lateral epicondyle of the humerus based on a search of PubMed and Web of Science databases. The literature provides ample information on the prevalence of complaints of pain in the lateral elbow region and its pathogenesis. However, there is no consensus among the authors about the recommended therapeutic procedure. Many treatment options have been proposed. Below, we present those that are most commonly used and have been described as being the most effective.

\section{Physical therapy}

Various forms of physical treatment are successfully used to reduce discomfort in patients suffering from the lateral elbow pain syndrome. Properly selected treatments stimulate collagen production, improving in this way, the strength and quality of the tendons. Comprehensive physical therapy also improves vascularization of the affected area. In other words, appropriately selected physical interventions stimulate repair processes in degenerated tendons and their attachments.

\section{Home exercise programs}

Many authors propose that tennis elbow can be treated at home with the help of personalized exercise programs (HEP).

The goal of the exercises is to restore normal elasticity to the antagonist groups of muscles (flexors and extensors) acting at the wrist and finger joints. The exercises consist in stretching these muscles. Exercise programs must be carefully selected to match the patient's current muscle condition. The exercises must also be skillfully introduced so that they do not exacerbate or renew the injury. Before a therapeutic session starts, the forearm and wrist should be placed in an unstressed position. This recommendation is sometimes mistakenly interpreted as meaning that the painful area should be immobilized. Immobilization, however, reduces resting muscle tension, weakens muscle strength, and impairs blood circulation, ultimately prolonging rehabilitation. Ice massage is recommended after each series of exercises,.

Pain resolves after six weeks of properly performed exercises. It is a mistake to stop rehabilitation at this point, however. The patient should continue exercising for up to 12 weeks, as discontinuation increases the risk of rapid recurrence. After 12 weeks, the frequency of the exercises should be reduced to two-three times a week, as a maintenance treatment.

Raman claims that resistance exercises can effectively reduce pain and improve forearm function, including grip strength. While this is true, the author does not define the optimal dosage of intervention. Other studies also provide evidence that the inclusion of eccentric exercises as part of a multimodal therapy program improves treatment outcomes in patients with tennis elbow.

\section{Ultrasounds and phonophoresis}

Ultrasound treatment involves the use of vibrations with a frequency above 20,000 Hz. During the procedure, an ultrasound probe emits mechanical energy and transmits it to the target tissue to a depth that depends on the emission wavelength. When applied to muscles, ultrasounds reduce their tension and relieve pain. During the procedure, blood vessels dilate and the inflammatory processes 
are inhibited and lose their dynamics. The procedure begins with applying a coupling agent, such as paraffin, ointment, gel or cream, over the painful area. A transducer, which generates a targeted ultrasonic wave, is applied to the treatment zone prepared in this way. The coupling agent may be inert or contain ingredients with analgesic or anti-inflammatory properties, in which case the procedure is called phonophoresis. The ultrasonic wave facilitates the penetration of the medicinal agent into diseased tissues with much greater efficiency than manual application of ointments or gels.

Treatment sessions usually take from one up to fifteen minutes. Patients are referred for a series of 12 to 20 treatment sessions. The number of sessions usually depends on the intensity of pain. However, strict compliance with general and local contraindications for the use of ultrasonic waves is mandatory.

\section{Cryotherapy}

Cryotherapy is the local or general use of low temperatures in therapeutic treatment. Local cryotherapy consists in reducing the temperature of the skin and deeper tissues in the affected area with the help of applicators that deliver controlled cooling to this area. As an effect of the local reduction of temperature, cell cytoplasm may freeze. The phase change of the semi-liquid cell content into a solid can lead to the rupture of the biological membranes and the destruction of the affected tissue. Under milder hypothermia, cell metabolism is slowed down and disease activity is inhibited. In tennis elbow, cold therapy helps to create conditions for further rehabilitation by reducing the secretion of pain mediators. As the edema is eliminated and muscles become more elastic, the patient can engage in limited physical activity. According to some research findings, cryotherapy can increase the rate of regeneration of damaged tissues. The literature clearly indicates that cold therapy is a useful treatment option for patients suffering from tennis elbow.

\section{Light therapy}

Irradiation of the painful region with lamps that emit various forms of visible and invisible light is widely used in physical therapy for musculoskeletal conditions and injuries. Polarized light induces a number of positive changes in treated tissues, leading to pain relief. This contributes to the regeneration of damaged collagen fibers, including those within and around the elbow joint. Such changes are due to improved blood supply caused by vasodilation and better perfusion of circulating blood. One treatment session takes about 20 minutes. It is important that the therapy be used regularly. In the treatment of the tennis elbow syndrome, sessions should be repeated regularly several times a day for at least two to three weeks.

\section{Laser therapy}

Laser therapy is a physical modality that uses a focused light beam. In physical therapy, two types of light with different wavelengths are used: infrared with a wavelength of $1100 \mathrm{~nm}$ and visible red with a wavelength of about $633 \mathrm{~nm}$. Laser beams at these wavelengths exert a biostimulating effect. They are used to treat a variety of lesions, including enthesopathy of the extensor muscles attached to the lateral epicondyle of the humerus. Laser therapy is very popular because the procedure does not present any technical problems, is fast, painless, highly effective, and poses no risk of infection. It is also important that laser therapy can be used in patients of all ages. With regard to the treatment of tennis elbow, a valuable feature of laser therapy is that it can be used in chronic, sub-acute and acute cases. Laser therapy has such a wide range of applications because it exerts multi-directional 
effects on tissues, in particular it shows anti-edematous, analgesic and anti-inflammatory activities. All these effects are obtained by activating microcirculation. Therefore, regardless of the severity and duration of the symptoms, favorable treatment outcomes are usually observed.

Morimoto, who conducted research on low-level laser therapy (LLLT), rated the effectiveness of this method at $66 \%$.

\section{Radial shock wave therapy}

For tennis elbow cases that have lasted more than 6 months and have not responded to other nonoperative procedures, some authors recommend ultrasound shock wave therapy (RSWT).

Radial shock waves are generated by means of a pneumatic system. RSWT has an analgesic effect and stimulates autoregeneration of the affected tissue by accelerating micro-circulation and cellular metabolism without damaging the adjacent tissues. In the case of tennis elbow, the average number of treatment sessions is five to eight every seven days. Intervals shorter than that are not recommended as the tissues subjected to the therapy require a sufficiently long time to regenerate. Experiences of pain during RSWT differ from patient to patient. Hence, therapists should take special care in selecting a suitable dosage for each patient. Kinesitaping of the elbow joint can be used as an adjunct to RSWT to promote relaxation of the tissues in the treated area.

\section{Pharmacotherapy and injections}

\section{Nonsteroidal anti-inflammatory drugs}

The most commonly used method of treating enthesopathy is anti-inflammatory and analgesic pharmacotherapy, which involves the administration of drugs of different chemical composition. Often, chronic administration of non-steroidal anti-inflammatory drugs (NSAIDs) leads to undesirable systemic side effects. Drugs which contain ketoprofen or diclofenac are now the drugs of choice, although they have not been demonstrated to have a specific effect on the function of fibroblasts or tenocytes .

Until recently, tendon attachment microinjuries were thought to cause enthesitis of the wrist extensor tendons. For this reason, NSAIDs have been used on a large scale. This therapeutic approach is still widespread. The latest microscopic and biochemical studies show, however, that damage to the tendon attachment in enthesopathies does not display a picture typical of inflammation. The dominant changes include alterations in the collagen structure and abnormal tendon blood supply at the enthesis site. In the light of these findings, tennis elbow, is not a tendinitis (an inflammatory process) but a tendinosis (a degenerative process).

This implies that the therapeutic effect of NSAIDs is analgesic. Therefore, it stands to reason that NSAIDs should be replaced by other pharmacotherapeutics.

\section{Nerve block injections - lidocaine and steroids}

Local infiltration with steroids and lidocaine is a routine treatment for tennis elbow, especially in patients in whom non-invasive treatment has failed to provide the expected resolution of symptoms. The painful site is palpated, and a ready-to-use steroid drug with lidocaine is administered locally at the tendon attachment site. This method of treatment has gained popularity, especially in sports medicine, because the injections produce a noticeable improvement, quickly relieving pain. Because the good analgesic effect is not permanent, the injections usually have to be repeated. Though this popular method of treatment produces instant results, it is not very effective in the long run and should not be recommended, especially in chronic forms of tennis elbow. The potential adverse effects, such as changes in skin tone and/or skin thinning as well as atrophy of subcutaneous fat and 
tendon and muscle fibers should all be taken into account.

It should be added that despite the fact that corticosteroid injections have a long record of use in the treatment of tendons, there is no scientific evidence of their beneficial effects, which makes their application controversial.

\section{Platelet-rich plasma}

Platelet-rich plasma is a concentration of platelets extracted from a patient's own blood and suspended in a small volume of plasma. It is obtained by centrifuging blood collected from the patient on an anticoagulant. During centrifugation, blood is separated into platelet-rich and plateletpoor plasma and red blood cells. Upon activation the thrombocytes in platelet-rich plasma release various growth factors, mainly platelet-derived growth factor (PDGF), transforming growth factor (TGF), vascular endothelial growth factor (VEGF) and epidermal growth factor (EGF), and cell adhesion proteins. The increased concentration of mediators of repair in the preparation promotes tissue regeneration. The mediators effectively stimulate the restoration of damaged tendons, muscles, ligaments, cartilage or bones. Because the injected material is autogenous, plasma treatment is safe and does not involve a risk of complications. The decision to use growth factors in the treatment of tendinopathy or enthesopathy must always be made by a physician based on the clinical picture and ultrasound findings of the tendon. The success of this therapy depends to a large extent on the tissue perfusion rate. In cases where perfusion is compromised as an effect of degenerative changes in the enthesis or where complete degeneration of blood vessels occurs, administration of platelet-rich plasma will be unfavorable. In such cases, surgery should be considered. Compared to steroid injections, the administration of autologous blood preparations shows much better short and medium-term therapeutic efficacy.

\section{Hyaluronic acid}

In clinical practice, local injection of hyaluronic acid derivatives has been observed to support the action of platelet-rich plasma in the treatment of enthesopathy of the lateral epicondyle of the humerus.

Hyaluronic acid has been found not only to contribute to inhibiting cartilage degeneration, but to have an analgesic effect. It is also believed that it can prevent degeneration of tendon tissue if treatment is started at an early stage of the disease.

\section{Botulinum toxin}

The myorelaxing effect of botulinum toxin has been used in the treatment of spastic paresis in patients with cerebral palsy. The reduction of muscle tone in the treatment of tendinopathy and enthesopathy of the lateral epicondyle of the humerus leads to a reduction in the constant pulling force at the attachment of the extensor carpi radialis brevis. Therefore, similarly to stretching exercises, this intervention has a pain relieving effect.

\section{Collagen}

Periarticular injections of collagen are an alternative to steroid, hyaluronic acid and platelet-rich plasma injections because they stimulate tissue repair and exert an analgesic effect.

\section{Surgery}

In patients who have experienced failure after 12 months of non-operative treatment of tennis elbow, a surgery should be considered. According to some authors, insufficient pain resolution and 
the inability to return to work after three months of treatment is an indication for an operative intervention.

Surgical options include open, percutaneous, and arthroscopic techniques. The primary goal of the intervention is to debride the affected extensor tendon tissue. In open surgery, a longitudinal incision is usually made over the lateral side of the elbow joint, gradually exposing the lateral epicondyle with the tendon attachment. Many different surgical approaches to enthesis repair have been proposed, and the choice of a repair technique depends on the intraoperative assessment of the morphology of the lesions. The solutions most frequently recommended in the literature include:

- $\quad$ release of the ECRB origin,

- fasciotomy of the common extensor origin,

- VY extensor tendon plasty,

- denervation of the lateral epicondyle,

- $\quad$ epicondylar resection with anconeus muscle transfer and lengthening of the ECRB.

Both arthroscopic and open surgical approaches produce good outcomes in the management of tennis elbow. They are safe and efficient solutions. However, a higher percentage of poor outcomes was found in patients who had undergone long-term conservative treatment prior to the surgery, especially treatment with steroid preparations.

Shahid found in his retrospective study of the outcomes of operative treatment of recalcitrant enthesopathy of the lateral epicondyle of the humerus that this method was highly effective in restoring the function and strength of the affected limb.

\section{Conclusions}

The wide gamut of available treatment options and their uncertain efficacy mean that there is no gold standard for the treatment of enthesopathy of the lateral epicondyle of the humeral bone. Treatment decisions are made individually by each orthopedist based on their knowledge, experience, and available treatment options. There is no evidence from large prospective clinical trials with long-term objective follow-up. Most studies report short- and medium-term treatment outcomes, evaluated according to different, subjectively selected criteria.

The prevailing view is that the elbow should be unloaded in the initial phase of treatment, often by immobilizing it in the functional position, with a recommendation of follow up physical therapy treatments administered in various configurations and often combined with pharmacotherapy, mainly with the use of anti-inflammatory drugs. The therapeutic protocol is modified depending on the treatment outcomes.

One important conclusion that follows from the state of the art in the treatment of tennis elbow is that the use of local corticosteroid injections should be limited. Although they can sometimes provide spectacular pain relief, the effect is short-lived, and, in cases of relapse, steroids predict poor prognosis for other forms of treatment.

Surgical treatment is reserved for patients in whom all safe forms of non-operative treatment have failed. 


\section{REFERENCES}

1. Shahid M, Wu F, Deshmukh SC. Operative treatment improves patient function in recalcitrant medial epicondylitis. Ann R Coll Surg Engl. 2013; 95(7): 486-488.

2. Morimoto Y, Saito A, Tokuhashi Y. Low level laser therapy for sports injuries. Laser Ther. 2013; 22(1): 17-20.

3. Raman J, MacDermid JC, Grewal R. Effectiveness of different methods of resistance exercises in lateral epicondylosis - a systematic review. J Hand Ther. 2012; 25(1): 5-25.

4. Salvi AE, Donini MT, Campochiaro G, Corona M, Dakovic I. Epicondylitis: etiology, pathogenesis and therapy. Tunis Med. 2011; 89(4): 320-325.

5. Levin D, Nazarian LN, Miller TT. et al. Lateral epicondylitis of the elbow: US findings. Radiology. 2005; 237(1): 230-234.

6. Maffulli N, Binfield PM, Moore D, King JB. Surgical decompression of chronic central core lesions of the Achilles tendon. Am J Sports Med. 1999; 27(6): 747-752.

7. Shiple BJ. How effective are injection treatments for lateral epicondylitis? Clin J Sport Med. 2013; 23(6): 502-503.

8. Wójcik G, Stawińska T, Szulc A, Sokołowska B. Diagnosis and conservative treatment of tennis elbow. Journal of Education, Health and Sport. 2016; 6(11): 98-107. 